\title{
A Bayesian Analysis in Determining Pro-rata Warranty Decision with Two-Dimensional Deterioration
}

\author{
Po-Chiang Tsai and Chih-Chiang Fang
}

\begin{abstract}
In practice, the condition of warranty should be taken into consideration for customers in order to get better post-sale service. Accordingly, manufacturers would use this as a marketing tool to advertise the quality of their products. However, offering warranty usually results in additional costs to the manufacturers. Therefore, the manufacturers would balance the costs and the benefits when they make the related decisions. Moreover, the deterioration of a product not only depends on time but also on usage. Under such situation, only considering one of them could misrepresent the estimation of products' deterioration, and therefore a two-dimensional failure model would be fit for dealing with such problems. Furthermore, most of the studies regarding pro-rata warranty issue mainly focused on cost analysis or reliability estimation but few studies would consider how the marketing strategies can be integrated into a synthetic decision. Another important issue is the manufacturer may not have sufficient historical data to estimate the deterioration of a newly developed product, and therefore the results obtained from analytical models may not be reliable. In order to deal with such a problem for the situation of insufficient historical data, a Bayesian analysis should be a reasonable approach. Accordingly, this paper proposes a Bayesian decision model which considers the pro-rata warranty with the pricing and the production strategy for the manufacturers.
\end{abstract}

Index Terms-Pro-rata warranty, repairable products, bayesian analysis.

\section{INTRODUCTION}

A product warranty is a contract offered by a manufacturer to its customers to repair or replace faulty items. From the buyer's perspective, the main role of a warranty is to safeguard the transaction. On the other hand, from the producer's perspective is a promotional instrument, similar to the price and performance of a product, to be used in competing with other manufacturers in the marketplace. However, it should be noted that offering unlimited warranty, with the intent of monopolizing the market, is unrealistic, since the costs will eventually outstrip the profits. Therefore, an appropriate warranty is important to the manufacturer.

Generally speaking, there are two basic warranty policies provided by the manufacturers. They are the free replacement warranty (FRW), which charges consumers no fee during the term of warranty, and the pro-rata warranty (PRW), which charges the consumer a preset proportion of cost for each

Manuscript received March 10, 2015; revised May 29, 2015. This work was supported by the Ministry of Science and Technology in Taiwan, under Chih-Chiang Fang's Grant: MOST-103-2410-H-366-002.

Po-Chiang Tsai is with the Department of Information Management, Shu-Te University, Taiwan, R.O.C. (e-mail: fransky@stu.edu.tw).

Chih-Chiang Fang is with the Department of International Business and Trade, Shu-Te University, Taiwan, R.O.C. (e-mail: ccfang@stu.edu.tw). repair within the term of warranty. From a practical point view, the PRW is a popular warranty policy for relatively high-priced products, such as plant facilities and large scale machines. However, the more the warranty proportion and length, the higher the operational costs will get, and which will eventually turns the product into unprofitable.

Most of studies regarding PRW issue mainly focus on the cost analysis with the consideration of reliability. The studies include the following: Menke [1] evaluated the warranty cost for a non-repairable product under PRW by the assumption of exponential failure process. Balcer and Sahin [2] proposed a stochastic failure distribution for deriving the expected warranty cost in which the repair cost is paid by the manufacturer in accordance with the proportion of damage under PRW. Blischke and Murthy [3] derived the expected warranty cost and the profit for renewable and non-renewable PRW from the perspectives of sellers and buyers, respectively under the assumption of Weibull failure model. Wang et al. [4] stated that warranty cost is positively related to products' reliability. By improving the reliability of products, the expected warranty cost can be reduced. Jain and Maheshwari [5] propose a warranty model for the renewing PRW, in which the failure rate of units, cost of preventive maintenance and cost of replacement are assumed to be constant. The proposed model can determine the optimal number and optimal period for preventive maintenance after the expiry of the warranty. Wu et al. [6] developed a cost model to determine the optimal burn-in time and warranty length for non-repairable products under FRW/PRW policy. Based on the results of the analysis, the fully renewing combination FRW/PRW is always better than the fully renewing policy in terms of cost.

In some cases, the manufacturer does not have sufficient historical data to estimate the deterioration of a newly developed product, the results obtained from analytical models may not be reliable. In such situations, the Bayesian analysis is a reasonable approach to additionally take expert opinions into account for better decision making. Papazoglou [7] used a Bayesian decision analysis to solve the problem of reliability certification. Papazoglou's approach is based on the existing prior assessment of uncertainties and further information can be obtained by testing the components. Juang and Anderson [8] utilized a Bayesian approach to determine an optimal adaptive preventive maintenance policy with minimal repairs.

Due to the fact that the deterioration of a product depends not only on time but also on usage. Under such situation, only considering one of them could misrepresent the estimation of equipment's deterioration. Therefore, two-dimensional failure model would be fit for dealing with such problems. Huang and Yen [9] provided manufacturers the guidelines 
that how to offer customers two-dimensional warranty programs with proper time and usage limits. Shahanaghi et al. [10] designed a two-dimensional extended warranty contract for automobile-manufacturers.

In this paper, we therefore proposed a mathematical model to help the manufacturer in systematically deciding pricing, production and warranty policy under insufficient information. The failure times of the product were assumed to be drawn from a non-homogeneous Poisson process (NHPP) with a specific intensity function. In the next section, we state and formulate the problem of the proposed model.

\section{ONE-DIMENSIONAL WARRANTY MODEL}

In this paper, the more general case of non-renewable products is focused, and the warranty policy under considerations is PRW. It is of special interest under the policy of PRW that the warranty proportion and term is a difficult trade-off, since the less the warranty proportion and the shorter warranty term are, the more cutback will be in the warranty cost, on the contrary, the more the warranty proportion and the longer warranty term are, the more attractive the product could be, and which may result in more marketing sales. In such case, the number of breakdowns or failures during the warranty term becomes crucial in choosing the optimal proportion for PRW because each breakdown will increase the warranty cost due to the required repairs and replacements. In line with the prior work, we assume that the nonhomogeneous Poisson process (NHPP) can properly describe the breakdown process of the product based on the following assumptions: 1) the repaired product is as good as old (i.e., minimal repair); 2) the repair time is negligible (Ascher and Feingold [11]), and the intensity function of the deterioration NHPP is $\lambda(t)$ which characterizes the deteriorating behavior of the process. Huang and Bier [12] suggested that the power law intensity function of the form $\lambda(t)=\alpha \beta t^{\beta-1}$ is more flexible and manageable than other used model, such as linear, exponential, or other mixed type intensity functions to appropriately describe the deteriorating behavior of deteriorating systems. Besides, Huang and Bier proposed a natural conjugate prior distribution for a power law deteriorating model which can be facilitated to analytically discuss the aforementioned decision problem, whereas other approaches with more general forms of intensity function may not be capable of performing the similar analysis. Therefore, in this paper, we simply assume that the successive failure times of the repairable product is drawn from an NHPP with a power law intensity function.

Since the synthetic warranty decisions would involve both the revenue of sales and the cost of production, the manufacturer has to realize these issues and take them into account. The demand function extended by Glickman and Berger [13] is considered in this paper because the function is fairly validated in many related studies, and which is given by

$$
\begin{gathered}
Q(W, \rho, P)=\omega_{1} P^{-\psi_{1}}\left(\omega_{2}+\rho^{\psi_{3}} W\right)^{\psi_{2}}, \\
\omega_{1}>0, \omega_{2}>0, \psi_{1}>1 \\
0<\psi_{2}<1, \text { and } 0<\psi_{3}<1
\end{gathered}
$$

where $\omega_{1}$ is an amplitude factor, and $\omega_{2}$ is a constant to allow for nonzero demand without warranty. Note that Equation (1) implies that a lower price, a higher proportion of the duty, or a longer warranty period results in more sales. The parameters $\psi_{1}, \psi_{2}$, and $\psi_{3}$ are elasticity of the price, the term warranty, and the proportion of the manufacturer's duty respectively. The values of the parameters $\psi_{2}$ and $\psi_{3}$ are between 0 and 1 because the customers' marginal demand will decrease over the extending warranty period or the raising proportion of the duty. Besides, if the warranty proportion $\rho=1$, it represents the decision maker adopt FRW policy. The above parameters could be obtained by market survey and analysis done by the marketing department.

Besides, in order to reduce the frequency of repairs and increase customer satisfaction, the manufacturer should provide a preventive maintenance program to slow down the deterioration of the product throughout the warranty period. Generally, a preventive maintenance program of a deteriorating system is usual periodical and imperfect. Its frequency of repairs is fixed, and the degree of recovery is less than 1 over the warranty period. Therefore, the expected number of minimal repairs with the age reduction factor $\eta$ during the period $[0, \mathrm{~W}]$ under the time interval $\mathrm{h}$ between maintenance visits should be

$$
\begin{aligned}
& N_{R}(\alpha, \beta, W, h, \eta) \\
& =\sum_{k=1}^{\lfloor W / h\rfloor} \int_{(k-1) h}^{k h} \lambda(t-\eta k h) d t+\int_{\lfloor W / h\rfloor h}^{W} \lambda(t-\eta\lfloor W / h\rfloor h) d t
\end{aligned}
$$

where $\lfloor W / h\rfloor$ is the lower integer part of the number of times of maintenance within the warranty period $[0, \mathrm{~W}]$. $\sum_{k=1}^{\lfloor W / h\rfloor} \int_{(k-1) h}^{k h} \lambda(t-\eta k h) d t$ is the expected number of failures within the time period $[0,\lfloor W / h\rfloor h]$, and $\int_{\lfloor W / h\rfloor h}^{W} \lambda(t-\eta\lfloor W / h\rfloor h) d t$ is the surplus of the expected number of failures after the $\lfloor W / h\rfloor$ 'th maintenance.

Due to mechanical aging of the deteriorating system, the each maintenance cost will get higher and higher. Accordingly, the maintenance cost per unit during the warranty period is (according to the assumptions of Jayabalan and Chaudhuri [14])

$$
C_{M}\left(C_{F}, \tau, h, W\right)=\sum_{k=1}^{\lfloor W / h\rfloor} C_{F}(1+\tau k h)
$$

where $C_{F}$ denotes the basic value of each preventive maintenance, and $\tau$ denotes the annual increasing rate of the preventive maintenance cost. Owing to that the PRW policy is adopted, the repair cost of the manufacturer's duty should be $\rho E\left[\mathrm{C}_{R}\right] N_{R}(\alpha, \beta, W, h, \eta)$.

From all the above discussions, the expected profit can be formulated as follows: 


$$
\begin{aligned}
\operatorname{Max} & \pi(W, \rho, P, Q) \\
= & P Q-C_{P} Q-\rho E\left[C_{R}\right] N_{R}(\alpha, \beta, W, h, \eta) Q \\
& \quad-C_{M}\left(C_{F}, \tau, h, W\right) Q
\end{aligned}
$$

Subject to: $Q=\omega_{1} P^{-\psi_{1}}\left(\omega_{2}+\rho^{\psi_{3}} W\right)^{\psi_{2}}$

Equation (4) include the total revenue of selling product, the total manufacturing cost $\left(C_{P}\right.$ : unit production cost), and the total warranty cost (the total repair cost and the total maintenance cost during the warranty period). Owing to that the warranty proportion and the warranty term should be discrete in practice (ex: $\rho=0.3,0.35,0.4, \ldots, 0.8 ; W=2,2.5$, $3, \ldots, 6)$, but most studies failed to recognize this fact by making an oversimplified assumption of continuity. Therefore, we need to enumerate the candidate warranty proportion and term under PRW policy ( $W_{i}, i=1 . . I ; \rho_{j}, j=1 . . J$ ) for deciding the optimal warranty proportion and term in practice. The optimal expected profit can be rewritten as follow:

$$
\begin{aligned}
\operatorname{Max}_{i, j} & \left\{\pi\left(W_{i}, \rho_{j}, P, Q\right)\right\}=P Q-C_{P} Q \\
& -\rho_{j} E\left[C_{R}\right] N_{R}\left(\alpha, \beta, W_{i}, h, \eta\right) Q \\
& -C_{M}\left(C_{F}, \tau, h, W_{i}\right) Q
\end{aligned}
$$

Subject to: $Q=\omega_{1} P^{-\psi_{1}}\left(\omega_{2}+\rho_{j}^{\psi_{3}} W_{i}\right)^{\psi_{2}}$

\section{TWO-DIMENSIONAL WARRANTY MODEL}

With regard to the two-dimensional failure model, we assume that the breakdown process of the product behaves bivariate NHPP process. Therefore, the failure intensity function is given as follow:

$$
\lambda(t, u)=\alpha_{1} \beta_{1} t^{\beta_{1}-1}+\alpha_{2} \beta_{2} u^{\beta_{2}-1}
$$

where the parameters $\alpha_{1}$ and $\beta_{1}$ represent the scale and shape parameters in terms of deterioration with time. The parameters $\alpha_{2}$ and $\beta_{2}$ represent the scale and shape parameters in terms of deterioration with usage. Therefore, without consideration of any PM policy, the expected number of minimal repairs of the bivariate model can be deduced as follow:

$$
\begin{aligned}
& \Lambda(t, u)=\int_{0}^{T_{0}} \int_{0}^{U_{0}} \lambda(t, u) \\
& =\alpha_{1} \beta_{1} t^{\beta_{1}-1}+\alpha_{2} \beta_{2} u^{\beta_{2}-1}=\alpha_{1} T_{0}^{\beta_{1}}+\alpha_{2} U_{0}^{\beta_{2}}
\end{aligned}
$$

The usage rate $s$ is a ratio of the using time to the usage $(s=t / u)$. In other word, it denotes the usage per unit time of a product. Due to the fact that the usage rate $\mathrm{s}$ is very helpful to transform the two-variate problem into univariate problem, we can utilize this to re-estimate the expected failures of products in consideration of all customers' usual practice. The Fig. 1 illustrates the relation between the using time and the usage for the usage rates.

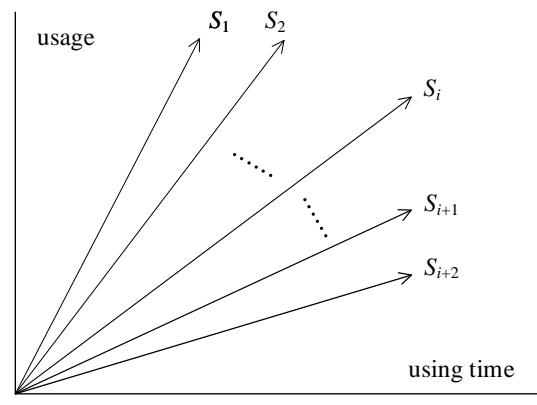

Fig. 1. The relation between the using time and the usage for the usage rates

Suppose that the usage rate $\mathrm{s}$ is a random variable and approximate to Gamma distribution according to all customers' usual practice, and therefore the estimation of the expected number of breakdowns should be rewritten as follow:

$$
\begin{aligned}
& \int_{0}^{T_{0}} \int_{0}^{U_{0}} \lambda(t, s) \times \operatorname{Gamma}(s \mid \theta, \gamma) d s d t \\
& =\int_{0}^{T_{0}} \int_{0}^{\infty}\left\{\alpha_{1} \beta_{1} t^{\beta_{1}-1}+s \alpha_{2} \beta_{2}(s t)^{\beta_{2}-1}\right\} \times \operatorname{Gamma}(s \mid \theta, \gamma) d s d t
\end{aligned}
$$

where $\operatorname{Gamma}(s \mid \theta, \gamma)$ is Gamma probability density function under the parameters $\theta$ and $\gamma$, and its form can be expressed as

$$
\begin{gathered}
\operatorname{Gamma}(s \mid \theta, \gamma)=\left(\frac{s^{\theta-1} e^{-s / \gamma}}{\gamma^{\theta} \Gamma(\theta)}\right) \\
\Gamma(\theta)=\int_{0}^{\infty} y^{\theta-1} e^{-y} d y
\end{gathered}
$$

Therefore, with consideration of preventive maintenance program, the expected number of minimal repairs with the age reduction factor $\eta$ during the period $[0, W]$ under the time interval $h$ between maintenance visits should be

$$
\begin{aligned}
& N_{R}\left(\alpha_{1}, \beta_{1}, \alpha_{2}, \beta_{2}, s, W, h, \eta\right) \\
& =\sum_{k=1}^{\lfloor W / h\rfloor} \int_{(k-1) h}^{k h} \int_{0}^{\infty}\left\{\begin{array}{l}
\alpha_{1} \beta_{1}(t-\eta k h)^{\beta_{1}-1} \\
+s \alpha_{2} \beta_{2}(s(t-\eta k h))^{\beta_{2}-1}
\end{array}\right\} \times \operatorname{Gamma}(s \mid \theta, \gamma) d s d t \\
& +\int_{\lfloor W / h\rfloor h}^{W} \int_{0}^{\infty}\left\{\begin{array}{l}
\alpha_{1} \beta_{1}(t-\eta\lfloor W / h\rfloor h)^{\beta_{1}-1} \\
+s \alpha_{2} \beta_{2}(s(t-\eta\lfloor W / h\rfloor h))^{\beta_{2}-1}
\end{array}\right\} \times \operatorname{Gamma}(s \mid \theta, \gamma) d s d t
\end{aligned}
$$

From the above discussion, the expected profit with consideration of two-dimensional warranty can be formulated as follows:

$$
\begin{aligned}
& \operatorname{Max}_{i, j}\left\{\pi\left(W_{i}, \rho_{j}, P, Q\right)\right\}=P Q-C_{P} Q \\
& -\rho_{j} E\left[C_{R}\right] N_{R}\left(\alpha_{1}, \beta_{1}, \alpha_{2}, \beta_{2}, s, W, h, \eta\right) Q \\
& -C_{M}\left(C_{F}, \tau, h, W_{i}\right) Q
\end{aligned}
$$

Subject to: $Q=\omega_{1} P^{-\psi_{1}}\left(\omega_{2}+\rho_{j}{ }^{\psi_{3}} W_{i}\right)^{\psi_{2}}$

The following assumptions should be noticed before you 
employ the model of the study:

1) The products' deteriorates due to time and usage, and the failure or breakdown process can be described by an NHPP.

2) A minimal repair is performed when a failure or breakdown occurs within the warranty period. After the repair, the product would be restored to its former status immediately.

3) The PM activities are imperfect so the condition of the product cannot fully restore the previous status after PM.

4) The probability distribution of the usage rate of the products is assumed to Gamma distribution.

\section{BAYESIAN ANALYSIS}

Suppose an NHPP with the power law intensity function can appropriately model the breakdown process. The optimal warranty proportion and term will be selected in accordance with the knowledge of the unknown parameters, which will be treated as random variables for Bayesian analysis, along with other considerations about costs from multiple perspectives.

Before proceeding with Bayesian analysis, the decision makers should verify that the assumptions are satisfied. The domain experts can provide the value of parameters $u_{\alpha}, \sigma_{\alpha}$, $u_{\alpha}$, and $\sigma_{\beta}$ according to prior knowledge. Besides, the market department would need to estimate the demand function of the new product based on market survey and analysis. After the above preparations, the decision makers can proceed with the prior analysis for the problem, and make the decision as long as they convinces the results of the prior analysis. If the decision makers consider that the prior analysis may not be very convincing and acceptable, the decision makers usually need more information to justify or correct their prior judgments. The posterior analysis can utilize few relevant data along with experts' opinions to refine the quality of the prediction.

For proceeding with the Bayesian analysis, the two formulations which respectively represent the optimal expected profit for the prior analysis and posterior analysis were constructed as follows:

Bayesian decision analysis is typically not easy to perform, since the derivation of posterior distributions might involve the use of numerical integration. Especially in our case of two random variables $(\alpha$ and $\beta$ ) in the state space, the analysis would be much more complicated to deal with. Huang and Bier (1998) proposed a natural conjugate prior distribution for the power law deteriorating model for repairable systems which is of the form

$$
f(\alpha, \beta)=K \alpha^{m-1} \beta^{m-1}\left(e^{-d} z^{m}\right)^{\beta-1} e^{-\alpha c z^{\beta}}
$$

where $K$ is the normalizing factor to ensure the distribution sums up to 1 . The major benefit of this natural conjugate prior distribution is to facilitate a straightforward analysis instead of the usually complicated computation. The four parameters in equation (3.8) (i.e. $c, d, m$, and $z$ ) can be chosen to achieve a suitable joint distribution with the desired prior marginal moments (the more details can refer to the study of Huang and
Bier (1998)). By using equation (12) as the prior distribution of $\alpha$ and $\beta$, it would be relatively easy to derive the prior mean of interest, and which is given by

$$
\begin{aligned}
& E\left[\alpha T^{\beta}\right]=\int_{A} \int_{B} \alpha T^{\beta} f(\alpha, \beta) d \beta d \alpha \\
& =\frac{m d^{m}}{c}(d+\ln [Z]-\ln [T])^{-m}
\end{aligned}
$$

where $T$ denotes an effective age of the deteriorate system. The prior analysis can be performed straightforwardly by calculating $E\left[\alpha T^{\beta}\right]$ in equations (13) with respect to the four parameters (i.e. c, d, m, and z) which are specified by the decision makers with their prior knowledge about the product $\left(u_{\alpha}, \sigma_{\alpha}, u_{\alpha}\right.$, and $\sigma_{\beta}$ ). The optimal prior decision is therefore determined without any complicated computation.

Suppose that the further investigation is undertaken, and the sample size $\mathrm{n}$ has been carefully examined. If the $n$ breakdown times (may from the other similar product) are collected and which are $\left(x_{1}, x_{2}, \cdots, x_{n}\right)$, then the posterior distribution can be effortlessly gained without further computation by the property of natural conjugate family, and which is given by

$$
\begin{aligned}
f^{\prime}(\alpha, \beta) & \propto L\left(D^{(n)} \mid \alpha, \beta\right) f(\alpha, \beta) \\
& =K^{\prime} \alpha^{m+n-1} \beta^{m+n-1}\left(e^{-d} z^{m} \prod_{i=1}^{n} x_{i}\right)^{\beta-1} e^{-\alpha\left(c z^{\beta}+x_{n}{ }^{\beta}\right)}
\end{aligned}
$$

However, the expectation of failures for the posterior analysis cannot be obtained in close form expression because the posterior distribution in Equation (14) is much more complex than the prior distribution in Equation (12). Fortunately, numerical integration can be employed to calculate the expectation of failures for the posterior analysis, which is given by

$$
\begin{aligned}
& E^{\prime}\left[\alpha T^{\beta}\right] \\
& =\int_{A} \int_{B} \alpha T^{\beta} K^{\prime} \alpha^{m+n-1} \beta^{m+n-1}\left(e^{-d} z^{m} \prod_{i=1}^{n} x_{i}\right)^{\beta-1} e^{-\alpha\left(c z^{\beta}+x_{n}^{\beta}\right)} d \beta d \alpha
\end{aligned}
$$

The posterior analysis can therefore be carried out with the considerations of both prior knowledge and sampling information.

\section{CONCLUSION}

The objective of this paper is to provide a methodology to solve the synthesized decision which concerns both warranty and marketing perspectives under the situation of scarce historical data. In the past, few studies discussed how the warranty proportion influences the related costs and profits so that the manufacturer might not take the advantage of the 
information to make a better decision. The future work could also be performed on refining the proposed model by considering the repair cost as a function of age, since the repair cost is subject to the degree of mechanical aging. A compound NHPP might be suitable for this analysis. It would be interesting to consider that the warranty proportion is subject to change as time elapses. However, such analysis would be expected to be more complicated to perform.

\section{ACKNOWLEDGMENT}

This work was supported by the Ministry of Science and Technology in Taiwan, under Chih-Chiang Fang's Grant: MOST-103-2410-H-366-002.

\section{REFERENCES}

[1] W. W. Menke, "Determination of warranty reserves," Management Science, vol. 15, pp. 542-549, 1969.

[2] Y. Balcer and I. Sahin, "Replacement costs under warranty: Cost moments and time variability," Operations Research, vol. 34, no. 4, pp. 554-559, 1986.

[3] W. R. Blischke and D. N. P. Murthy, "Warranty cost analysis," Marcel Dekker, New York, 1994.

[4] L. H. Wang, K. Suzuki, and W. Yamamoto, "Age-based warranty data analysis without date-specific sales information," Applied Stochastic Models in Business and Industry, vol. 18, issue 3, pp. 323-337, 2002.

[5] J. Madhu and M. Sandhya, "Discounted costs for repairable units under hybrid warranty," Applied Mathematics and Computation, vol 284, pp. 914-920, 2006.

[6] C. C. Wu, C. Y. Chou, and C. K. Huang, "Optimal burn-in time and warranty length under fully renewing combination free replacement and pro-rata warranty," Reliability Engineering and System Safety, vol. 92, pp. 887-901, 2007.

[7] I. A. Papazoglou, "Bayesian decision analysis and reliability certification," Reliability Engineering and System Safety, vol. 66, pp. 177-198, 1999.

[8] N. Juergen, "Comparing quality signals as tools of consumer protection: Are warranties always better than advertisements to promote higher product quality," International Review of Law and Economics, vol. 24, pp. 227-239, 2004.
[9] Y. S. Huang and C. Yen, "A study of two-dimensional warranty policies with preventive maintenance," IIE Transactions, vol. 41, no. 4, pp. 299-308, 2009

[10] K. Shahanaghi et al., "Failure modeling and optimizing preventive maintenance strategy during two-dimensional extended warranty contracts," Engineering Failure Analysis, vol. 28, pp. 90-102, 2013.

[11] H. Ascher and H. Feingold, "Repairable systems-modeling, inference, misconceptions and their causes," Marcel Dekker, New York, 1984

[12] Y.-S. Huang and V. M. Bier, "A natural conjugate prior for the nonhomogeneous poisson process with a power law intensity function," Communications in Statistics-Simulations and Computation, vol. 27, pp. 525-551, 1998.

[13] T. S. Glickman and P. D. Berger, "Optimal price and protection period for a product under warranty," Management Science, vol. 22, pp. 1381-1390, 1976

[14] V. Jayabalan and D. Chaudhuri, "Cost optimization of maintenance scheduling for a system with assured reliability," IEEE Transactions on Reliability, vol. 41, pp. 21-25, 1992.

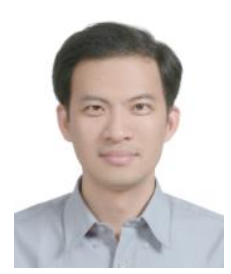

Po-Chiang Tsai is currently a lecturer in the Department of Information Management at Shu-Te University, Taiwan, R.O.C. He earned his M.S. degree from the Department of Computer Science at Stevens Institute of Technology, U.S. His research interests include decision analysis, database, and soft computing.

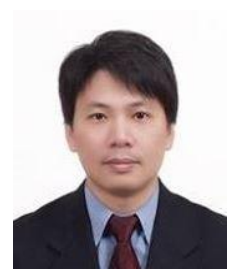

Chih-Chiang Fang is currently an associate professor in the Department of Information Management at Shu-Te University, Taiwan, R.O.C. He earned both his M.S. and Ph.D. degrees from the Department of Industrial and Information Management at National Cheng Kung University. His research interests include decision analysis, data mining, and software engineering. Related papers have appeared in such professional journals, such as Naval Research Logistics, IEEE Transactions on Engineering Management, Software Testing, Verification and Reliability, Computers \& Industrial Engineering, International Journal of Production Economics, International Journal of Production Research, Decision Support Systems and others. 